\title{
A Review on Prediction of Heart Disease Diagnosis based on ANFIS Model
}

\author{
Miss. Shraddha R. Warhade ${ }^{1}$, Prof. U.W. Hore ${ }^{2}$ \\ Student, Dept of Electronics and Telecommunication Engineering, P.R. Pote (Patil) College of Engineering and \\ Management, Amravati, India ${ }^{1}$ \\ Lecturer, Dept of Electronics and Telecommunication Engineering, P.R. Pote (Patil) College of Engineering and \\ Management, Amravati, India ${ }^{2}$
}

\begin{abstract}
Cardiovascular diseases have become the main cause of death in most of the countries of the world. The considerable growing of cardiovascular disease and its effects and complications as well as the high costs of treatment makes medical community seek for solutions to prevention, early identification and effective treatment with lower costs. Thus, valuable knowledge can be established by using artificial intelligence, the discovered knowledge makes improve the quality of service. Heart disease is a term assigned to a large number of medical conditions related to heart. These medical conditions describe the abnormal health conditions that directly influence the heart and all its parts. Heart disease is a major health problem in today's time. Technically, the ANFIS performs a vital role for prediction of diseases in medical industry. Diagnosis of heart disease by using machine learning methods is one of the challenges in the health field. This project will be provided on a particular dataset using classification and feature selection approach. In this we will use feature ranking on effective factors of disease related to Cleveland clinic database and by using ANFIS, 13 effective factors reduced in terms of cost and accuracy.
\end{abstract}

Keywords: heart disease diagnosis, ANFIS, classification, feature selection.

\section{INTRODUCTION}

Life is dependent on efficient working of heart because heart is essential part of our body. If operation of heart is not proper, it will affect the other body parts of human such as brain, kidney etc. Heart disease is a disease that affects on the operation of heart. Heart and blood vessel disease are also called heart disease which includes numerous problems, many of which are related to a process called atherosclerosis. Atherosclerosis is a condition that develops when a substance called plaque builds up in the walls of the arteries. This build up narrows the arteries, making it harder for blood to flow through. If blood clots formed, it can stop the blood flow. This can cause a heart attack or stroke. There are number of factors which increases risk of heart disease. High blood cholesterol and high triglyceride levels, high blood pressure, diabetes and pre diabetes, overweight and obesity, smoking, Lack of physical activity, unhealthy diet and stress.

Nowadays, in the world, Heart disease is the major cause of deaths which is also supported by the World Health Organization survey which has estimated that 12 million deaths occur worldwide, every year due to the Heart diseases. Half the deaths in the United States and other developed countries occur due to cardio vascular diseases. It is also the chief reason of deaths in numerous developing countries. On the whole, it is regarded as the primary reason behind deaths in adults. Heart disease kills one person every 34 seconds in the United States.
Eminent health services imply diagnosing patients correctly and providing effective treatment. Inferior medical decisions can lead to disastrous consequences which are not acceptable. Health industry must also try to minimize the number of tests for identifying the disease. All this can be achieved by commissioning appropriate decision support system. Today many healthcare organizations have employed hospital information systems for managing patient's data. These systems were built for supporting billing, inventory management and for statistical calculations.

Unfortunately this data is not used for decision making. This huge data can be used for answering questions like "predict the probability of patients getting heart disease". Medical diagnosis is regarded as an important yet complicated task that needs to be executed accurately and efficiently.

The automation of this system would be extremely advantageous. Regrettably all doctors do not possess expertise in every sub specialty and moreover there is a shortage of resource persons at certain places. Therefore, an automatic medical diagnosis system would probably be exceedingly beneficial by bringing all of them together.

In table I main features for heart attack diagnosis related to UCI Dataset are shown along with their types. 
Vol. 5, Issue 12, December 2016

TABLE I. MAIN FEATURES FOR HEART ATTACK DIAGNOSIS

\begin{tabular}{|l|l|l|}
\hline S.N & Attribute Name & Description \\
\hline 1 & Age & Age in years \\
\hline 2 & Sex & Male=1, Female=0 \\
\hline 3 & Cp & Chest pain type \\
\hline 4 & Trestbps & $\begin{array}{l}\text { Resting Blood pressure upon hospital } \\
\text { nadmission }\end{array}$ \\
\hline 5 & Cholestrol & Serum Chol \\
\hline 6 & FBS & Fasting blood sugar $>120$ (true=1, false=0) \\
\hline 7 & RestingECG & Resting Electrocardiographic Results \\
\hline 8 & Thalach & Maximum Heart Rate \\
\hline 9 & Exang & $\begin{array}{l}\text { Does the patient experience angina as a result } \\
\text { of exercise (value 1:yes, value 0:no) }\end{array}$ \\
\hline 10 & Old peak & $\begin{array}{l}\text { ST depression indeced by exercise relative to } \\
\text { rest }\end{array}$ \\
\hline 11 & Slope & Slope of the peak exercise \\
\hline 12 & Thal & $\begin{array}{l}\text { Value 3: Normal, value 6: fixed defect, value } \\
7: \text { reversible defect }\end{array}$ \\
\hline 13 & CA & $\begin{array}{l}\text { Number of major vessels colored by } \\
\text { fluoroscopy (value 0-3) }\end{array}$ \\
\hline 14 & ConceptClass & Angiographic disease status \\
\hline
\end{tabular}

Appropriate computer-based information and/or decision support systems can aid in achieving clinical tests at a reduced cost. The purpose of this research is to develop a model for prediction based on the demographic data of the patients' and provide best accurate result using ANFIS (adaptive neuro fuzzy interference system). In this we will use feature ranking on effective factors of disease related to Cleveland clinic database and by using ANFIS, 13 effective factors reduced in terms of cost and accuracy.

\section{LITERATURE REVIEW}

In this paper we survey different papers in which one or more method used for prediction of heart disease. The related work consists of attributes containing patient's medical history and symptoms. Majid Ghonji Feshki, Omid Sojoodi Shijani [1] proposed the improving the heart disease diagnosis by evolutionary algorithm of pso and feed forward neural network. In this research the work has been provided on a particular dataset using classification and feature selection approach.and provide the best accurate criteria for heart dieses diagnosis. The assessment of selected features of classified methods also showed that PSO method along with Neural Networks of Feed Forward Back-Propagation has the best accurate criteria.

Beant Kaur,Williamjeet Singh[2] proposed review on heart disease prediction system using data mining techniques. Data mining provides the methodology and technology to transform mounds of data into useful information for decision making and use this data for the prediction of heart disease with good accuracy. So that the prediction by using data mining algorithm given efficient results. Applying data mining techniques to heart disease treatment data can provide as reliable performance as that achieved in diagnosing heart disease.

Jyoti Soni ,Ujma Ansari, Dipesh Sharmah,Sunita Soni[4] proposed predictive data mining for medical diagnosis. the problem of constraining and summarizing different algorithms of data mining used in the field of medical prediction are discussed. The focus is on using different algorithms and combinations of several target attributes for intelligent and effective heart attack prediction using data mining. They conclude that the accuracy of the Decision Tree and Bayesian Classification further improves after applying genetic algorithm to reduce the actual data size to get the optimal subset of attribute sufficient for heart disease prediction.

K.Prasanna Lakshmi, Dr. C. R .K.Reddy [3] proposed fast rule-based heart disease prediction using associative classification mining. This research uses associative classification which builds a classifier with prediction rules of high interestingness values using Associative Classification and rewarding approach and experimental results show that this work helps doctors in their diagnosis decisions.

The most common methods used by researchers for diagnosis and monitoring of cardiovascular disease are classification and clustering methods. However, what has been less into the attention so far is the investigation of the issue by several types of methods and reaching a consensus in order to diagnose heart disease with highest precision and availability in the shortest time and the lowest cost as possible. Therefore, the purpose of the present study was to select the best features with the lowest costs and shortest times and highest precisions using ANFIS classification model.

An easy way to comply with the conference paper formatting requirements is to use this document as a template and simply type your text into it.

\section{METHODOLOGY}

Based on the principle of ANFIS, this research have six processes initiated by data collection, attribute and data selection, data preprocessing, data resampling modeling, and evaluation. The scope of method in this research has been shown in Fig. 1.

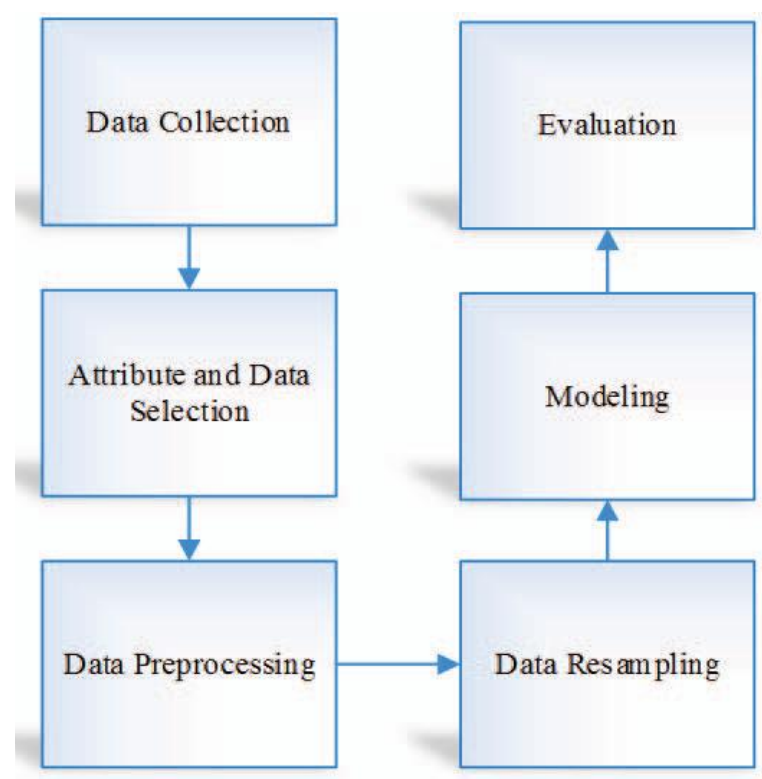

Fig 1. Flow methodology of work. 
Vol. 5, Issue 12, December 2016

An adaptive neuro fuzzy inference system or adaptive network based fuzzy inference system (ANFIS) is a kind of artificial neural network that is based on TakagiSugeno fuzzy inference system. Since it integrates both neural networks and fuzzy logic principles, it has potential to capture the benefits of both in a single framework. Its inference system corresponds to a set of fuzzy IF-THEN rules that have learning capability to approximate nonlinear functions. For using the ANFIS in a more efficient and optimal way, one can use the best parameters obtained by genetic algorithm.

In health, the data is mostly extracted from the client files at health centers. To conduct this research the data related to Cleveland clinic existing in UCI machine learning repository were used. This dataset has collected the data related to 303 healthy and sick people from which 138 were sick and 165 were healthy. This database contains 76 attributes, but all publications refer to using a subset of 14 of them, which 13 are related to disease factors and 1 case is related to the presence or absence of heart disease in people who underwent 13 previous tests. These attributes shown in below table. It must be noted that these factors are in fact the most important and most basic factors ever identified to diagnose heart attacks; therefore the investigation on these factors can be considered quite precise and important. Using a given input/output data set, the toolbox function ANFIS constructs a fuzzy inference system (FIS) whose membership function parameters are tuned (adjusted) using either a backpropagation algorithm alone, or in combination with a least squares type of method.

\section{CONCLUSION}

Diagnosis of heart disease by using machine learning methods is one of the challenges in the health field. The purpose of this research is to develop a model for prediction based on the demographic data of the patients' and provide best accurate result using ANFIS (adaptive neuro fuzzy interference system). In this we will use feature ranking on effective factors of disease related to Cleveland clinic database and by using ANFIS, 13 effective factors reduced to optimized features in terms of cost and accuracy.

\section{REFERENCES}

[1] Majid Ghonji Feshki, Omid Sojoodi Shijani proposed the Improving the Heart Disease Diagnosis by Evolutionary Algorithm of PSO and Feed Forward Neural Network ,2016

[2] Beant Kaur, Williamjeet Singh proposed Review on Heart Disease Prediction System using Data Mining Techniques. International Journal on Recent and Innovation Trends in Computing and Communication, 2014

[3] K.Prasanna Lakshmi, Dr. C. R .K.Reddy proposed Fast Rule-Based Heart Disease Prediction using Associative Classification Mining. IEEE International Conference on Computer, Communication and Control (IC4-2015)

[4] Jyoti Soni ,Ujma Ansari, Dipesh Sharmah,Sunita Soni proposed Predictive Data Mining for Medical Diagnosis: An Overview of
Heart Disease Prediction. International Journal of Computer Applications (0975 - 8887)Volume 17- No.8, March 2011

[5] N. Aditya Sundar1, P. Pushpa Latha2, M. Rama Chandra3 proposed Performance analysis of classification data mining techniques over heart disease data base. International journal of engineering science \& advanced technology

[6] Teerapat Kansadub1,Sotarat Thammaboosadee2, Supaporn Kiattisin3 proposed stroke risk prediction model based on demographic data. The 2015 Biomedical Engineering International Conference

[7] Panday, P. and N. Godara, Decision support system for cardiovascular heart disease diagnosis using improved multilayer perception. International Journal of Computer Applications, 2012. 45(8): p. 12-20.

[8] Subbalakshmi, G., K. Ramesh, and M.C. Rao, Decision support in heart disease prediction system using naive bayes. Indian Journal of Computer Science and Engineering (IJCSE), 2011. 2(2): p .170176.

[9] Abdullah, A. and R. Rajalaxmi. A data mining model for predicting the coronary heart disease using random forest classifier. in International Conference in Recent Trends in Computational Methods, Communication and Controls. 2012.

[10] Kumari, M. and S. Godara, Comparative study of data mining

[11] Classification methods in cardiovascular disease prediction. 2011.

[12] Srinivas, K., B.K. Rani , and A. Govrdhan, Applications of data mining techniques in healthcare and prediction of heart attacks. International Journal on Computer Science and Engineering (IJCSE), 2010. 2(02): p. 250-255.

[13] Abdullah, A. and R. Rajalaxmi. A data mining model for predicting the coronary heart disease using random forest classifier. in International Conference in Recent Trends in Computational Methods,Communication and Controls. 2012.

[14] Soni, .I., et aI., Intelligent and effective heart disease prediction system using weighted associative classifiers. International Journal on Computer Science and Engineering, 2011. 3(6): p. 2385-2392.

[15] Khemphila, A. and V. Boonjing. Heart disease classification using neural network and feature selection. in Systems Engineering (ICSEng), 2011 21st International Conference on. 2011. IEEE.

[16] Nahar, .I., et aI., Computational intelligence for heart disease diagnosis: A medical knowledge driven approach. Expert Systems with Applications, 2013. 40(1) :p. 96-104. 